\title{
Phoneme-monitoring reaction time as a function of preceding intonation contour
}

\author{
ANNE CUTLER \\ Massachusetts Institute of Technology, Cambridge, Massachusetts 02139
}

\begin{abstract}
An acoustically invariant one-word segment occurred in two versions of one syntactic context. In one version, the preceding intonation contour indicated that a stress would fall at the point where this word occurred. In the other version, the preceding contour predicted reduced stress at that point. Reaction time to the initial phoneme of the word was faster in the former case, despite the fact that no acoustic correlates of stress were present. It is concluded that a part of the sentence comprehension process is the prediction of upcoming sentence accents.
\end{abstract}

The greater the degree of stress assigned to a word in a sentence, the longer its vowel (or the vowel of its stressed syllable) will be, the higher the relative pitch of the word will be, and, in general, the greater the peak amplitude of its stressed syllable will be (Lehiste, 1970). Words assigned little relative stress in a sentence, on the other hand, tend to have shorter duration, lower relative pitch, and less relative amplitude; further, the vowels in unstressed words of ten reduce to $/ \mathrm{\partial} /$. It is reasonable to expect that words that are longer, louder, and higher pitched should be somewhat easier to comprehend than words that are shorter, softer, and lower pitched and contain reduced vowels; indeed, Lieberman (1963) has shown that this is true of isolated words which are excised from a sentence context. Such differences in ease of comprehension, or intelligibility, should be of importance in the course of sentence processing. The more easily an acoustic representation can be decoded, the more quickly its meaning can be retrieved from the mental lexicon to contribute to the semantic representation being constructed of the sentence.

That assigning stress to an item in a sentence will affect the role that item takes in the comprehension process has, in fact, been demonstrated by several recent investigations using the phoneme monitoring technique. In a phoneme monitoring experiment, subjects are asked to comprehend sentences and at the same time to listen within them for the occurrence of a specified word-initial phoneme. Reaction time to the target phoneme in this task has been assumed to be sensitive to momentary processing difficulty during sentence comprehension; it is raised, for instance, by the occurrence immediately prior to the target-bearing word of a low-frequency word (Foss, 1969) or of an ambiguous item (Foss, 1970). Cutler and Foss (in press), investigating the source of faster reaction times to

This study formed part of a dissertation presented to the Graduate School of the University of Texas at Austin. The author is very grateful to Dr. Donald J. Foss for advice and encouragement. Financial support was provided by National Institute of Education Grant NEG-00-3-0120 to Donald J. Foss. content words (nouns and verbs) than to function words (prepositions, conjunctions) in prior phoneme monitor ing experiments, demonstrated that this difference resulted from the higher level of stress assigned to content words in the sentence, rather than from any intrinsic advantage due to form class; when stress was controlled, the reaction time advantage of content words disappeared. Shields, McHugh, and Martin (1974) measured reaction time to targets on nonsense words such as "benkik," which were embedded in sentences in a location where a proper noun might be expected to occur; they found that reaction time to the same targets in the same sentences was faster when the nonsense word was stressed on the target-bearing syllable than when it was stressed on the second syllable.

It does not seem unreasonable to assume that this difference in processing complexity between stressed and unstressed words is due to the acoustic correlates of stress. Heightened intelligibility should lead to an increase in the speed with which the separate phonemes of the input can be identified, and hence in the speed with which the occurrence of a target phoneme can be acknowledged. However, there is evidence which indicates that to ascribe the entire difference to acoustic factors might be overhasty. The experiment by Shields et al. included a control condition in which the nonsense words were excised from the experimental sentences and presented to subjects for phoneme monitoring in a string of other nonsense words. Under these conditions, no reaction time difference was found between the nonsense words stressed on the target-bearing syllable and those stressed on the second syllable. Since the stimuli in the control task were identical to the experimental stimuli, Shields et al. concluded that the reaction time difference found with the experimental sentences was not due to acoustic variations.

They claimed, instead, that rhythmic cues in the sentences allowed hearers to predict the location of upcoming accents. Thus, they claimed that their result provided support for the theory proposed by Martin (1972), according to which the elements of a speech 
signal are temporally organized, the organization being entirely determined by the syllabic stress patterns of the words comprising the utterance. Listeners may utilize these strong rhythmic cues by "locking" onto the rhythmic pattern of a sentence as early as possible; if this is successful, they are then able to predict the location of stressed items in the later parts of the sentence.

The hypothesis that listeners make such predictions constitutes a fairly strong claim, and the results from the control condition in Shields et al.'s experiment provide only slim evidence on which to base it. The processing of a string of nonsense words may, for instance, be a task so foreign to subjects that the advantage of greater intelligibility of stressed syllables is simply masked by an overall rise in the level of difficulty. Support for this suggestion can be found in the fact that the mean reaction times in Shields et al.'s control sequences averaged $100 \mathrm{msec}$ longer than the mean reaction times to targets in the sentences.

The claim that the sentence processing mechanism engages in active prediction of upcoming stress locations is, however, a very interesting one; certainly, it carries weightier implications for models of the sentence comprehension process than does the counterclaim, that the reaction time difference between stressed and unstressed words is due entirely to the effects of heightened acoustic clarity. A more exacting test of the hypothesis is therefore in order, a test, moreover, in which normal sentence comprehension is better approximated than was the case in the experiment by Shields et al. A closer approximation can be achieved by presenting sentences rather than nonsense strings, with words rather than nonsense items bearing the phoneme target.

For instance, if the same (acoustically identical) word could be shown to elicit different reaction times depending on the suprasegmental contour in which it appeared, the reaction time difference could not be due to acoustic variation in the word itself. Two sentences which differ in suprasegmental contour and yet each contain a given word, acoustically identical in each occurrence, can be produced by tape-splicing. In the experiment to be described here, two copies of one recording of a word-i.e., two acoustically identical sequences-were spliced into two different contexts. These contexts consisted of the same words in each case, spoken, however, with two different intonation patterns. The sequence preceding the target item in the two contexts, therefore, was identical in every way except in intonation contour-the words did not differ, but the relative pitch, duration, and amplitude assigned to each word differed markedly.

The intonation pattern of the one context, intact, assigned high stress to the target-bearing item, the pattern of the other low stress. Once the original targetbearing word had been removed from each context and the acoustically identical replacements inserted, the one context predicted that the target-bearing item would carry high stress (that is, the preceding intonation was consonant with the occurrence in that position of a highly stressed item), while the other predicted that it would bear reduced stress (i.e., the preceding contour indicated that at that point stress would be reduced). If differences in intelligibility were solely responsible for the previous reaction time advantage of stressed words, no difference should be found between reaction times to the target in the two different contexts. If, however, part of sentence processing is the prediction of upcoming stress locations and the reaction time advantage is due at least in part to this, then we expect that the target word will produce faster reaction times when it is embedded in the context which indicates that it will bear high stress than when it is embedded in the context which indicates that its stress level will be low.

\section{METHOD}

\section{Materials}

Twenty unrelated sentences were recorded in three versions. In one version, the target-bearing word was heavily stressed. In the second version, that word received very reduced stress. In the third version, neutral, or intermediate, stress was assigned to the target word. In order to make the intonation contours sound natural, the three versions had different endings; however, the point at which the sentences diverged was beyond the occurrence of the target. The target-bearing word was, in each case, a monosyllabic noun beginning with one of the three phonemes used as targets $-/ \mathrm{b} /, / \mathrm{d} /, / \mathrm{k} /$-and it occurred more than five syllables after the beginning of the sentence, and not at the end of the sentence. $/ \mathrm{d} /)$ :

An example sentence is the following (phoneme target

High stress on target:

She managed to remove the dirt from the rug, but not the berry stains.

Low stress on target: She managed to remove the dirt from the rug, but not from their clothes.

Neutral version: She managed to remove the dirt from the rug.

As can be seen from the different endings, the relative increase and decrease in target item stress of the experimental versions in comparison with the neutral version has been obtained by manipulation of what is commonly called contrastive stress (Bolinger, 1961).

The target words were spliced out of all three versions of each sentence. The high- and low-stress target items were discarded, and copies of the target item from the neutral version of each sentence were spliced into their places in the first and second versions. The experimental sentences thus consisted of two versions of each sentence-one in which the intonation contour predicted high stress on the target-bearing word, and one in which the contour predicted low stress on that word. The target word itself was, however, intermediate in stress, and, moreover, identical in both versions.

Two tapes were constructed, each containing one version of each of the 20 experimental sentences plus 40 filler items, 20 of these without occurrence of the target. Predicted high vs. predicted low stress was counterbalanced across the two tapes for the experimental items. Two further tapes werc compiled, containing the same filler sentences plus the original high-and low-stress versions of the 20 experimental sentences, balanced across tapes for stress level. 


\section{Subjects}

The subjects were 208 undergraduates at the University of Texas who participated as part of a course requirement, and two secretaries employed at the University of Texas. A total of 63 subjects in three separate administrations heard each of the two spliced tapes, while 42 in two administrations heard each of the unspliced tapes.

\section{Procedure}

The subjects were tested in groups of up to six at a time. They were told that they were participating in an experiment on sentence comprehension, and were instructed to pay careful attention to the content of the sentences, since a comprehension test would be given at the end of the experiment. In addition, they were asked to press a button whenever they heard a word in a sentence beginning with the target phoneme specified for that sentence. The targets varied across sentences, and the particular target for each sentence was specified immediately prior to the presentation of the sentence. The sentences were spoken by a male speaker of standard American, and were presented binaurally over headphones. A timer was automatically started when the target occurred and was stopped by the subject's action in pressing the button. Reaction times shorter than $100 \mathrm{msec}$ or longer than $1,500 \mathrm{msec}$ were discarded, since it was felt that the former might result from anticipations, the latter from a reprocessing of the sentence. Timing and data collection were under the control of a PDP-8/I computer.

The comprehension test was administered immediately upon conclusion of the set of sentences. The subjects were asked to judge for each of a list of sentences, some of which had occurred in the experiment and some of which had not, whether or not they had heard it in the experiment.

A separate test was carried out to investigate the possible effects on the reaction time results of the acoustic discontinuity in the sentences introduced by the splicing intervention. While this discontinuity was small, it was nonetheless perceptible: it is possible that the neutral-stress word consistently produced a greater effect of oddness-i.e., the resulting intonation sounded more anomalous-when it was embedded in either a high-stress or a low-stress context, and that the reaction time differences reflected this oddness of the contour. A tape, consisting of the entire 40 spliced experimental sentences plus 20 other items, was therefore played to a group of 63 University of Texas undergraduates, none of whom had served as subjects in the experiment. The 20 additional items comprised 10 of the filler sentences from the experimental tapes, spoken with normal intonation, and 10 syntactically well-formed sentences made up of individual words or short phrases spliced together from separate recordings. The intonation of the lat ter sentences deviated from normal to a greater or lesser extent. Subjects were specifically instructed to attend to the intonation of the sentences and were asked to rate the "oddness" of each sentence's contour on a 5-point scale from "perfectly normal" to "extremely weird."

\section{RESULTS}

Performance on the comprehension test was good, indicating that subjects had indeed comprehended the sentences. The overall mean correct was $68 \%$ for the spliced and $70 \%$ for the unspliced versions.

A mean reaction time score for each subject for each condition was obtained; these means are presented in Table 1.

Two separate analyses of variance were performed on the reaction time data. As Clark (1973) has pointed out, both Subjects and Sentences should be treated as random factors, in order to assume that effects are generalizable beyond the particular samples of each used in
Table 1

Mean Reaction Time (Milliseconds) to Presence of Target Phoneme

\begin{tabular}{lccc}
\hline & Predicted & Predicted & \\
& High Stress & Low Stress & Mean \\
Spliced Sentences & 378 & 417 & 398 \\
& High Stress & Low Stress & \\
Unspliced Sentences & 294 & 403 & 349 \\
\hline
\end{tabular}

the study. Accordingly, one analysis was performed on the mean reaction time scores for each subject, collapsing across Sentences, while a second analysis was performed on the mean reaction time scores for each sentence, collapsing across Subjects. The combined results of these two analyses enabled the $\min F$ statistic to be computed. Since the experiment had been conducted three times with several months intervening between administrations, the means were computed separately for each occasion and a variable of Administration included in the analysis. Since this variable had unequal subject Ns in its separate instances, the analysis by Subjects was performed as an unweighted mean analysis (Winer, 1971).

In the spliced tapes, the effect of predicted stress level was significant, $\min F^{\prime}(1,30)=6.54, p<.025$, with predicted high-stress targets eliciting faster reaction times than predicted low-stress targets. The Administration variable was not significant and did not interact with the predicted Stress Level variable.

The additional variable of Materials Sets in the analysis by Subjects was not significant, and did not interact with any other variable.

In the unspliced tapes, the effect for stress alone was highly significant, $\min F^{\prime}(1,25)=18.22, p<.001$, again with high-stress targets eliciting faster reaction times. There was also a main effect for Administration on these control tapes, $\min F(1,74)=5.71), p<.025$, with reaction times on one administration being faster than those on the other.This effect did not, however, interact with Stress Level. In the analysis by Subjects, the main effect of Materials Sets was not significant and did not interact with Stress Level. It did, however, interact weakly with the Administration by Stress Level interaction, $F(1,80)=4.33, p<.05$; in the first administration, Tape 1 produced longer reaction times for high-stress items than did Tape 2, whereas in the second administration the reverse was the case. It is doubtful whether any importance should be attached to this finding.

The "oddness" ratings for the 20 spliced experimental sentences were scored as follows: each position on the scale was weighted, from 1 for "perfectly normal" through 5 for "extremely weird." A mean rating, collapsed across subjects and sentences, was computed for both predicted high- and predicted low-stress items, and for the two types of filler item. The mean ratings are given in Table 2.

An analysis of variance, collapsing across subjects, 
Table 2

Mean "Oddness" Ratings

\begin{tabular}{|c|c|c|c|}
\hline \multicolumn{2}{|c|}{ Experimental Sentences } & \multicolumn{2}{|c|}{ Filler Sentences } \\
\hline $\begin{array}{c}\text { Predicted High } \\
\text { Stress }\end{array}$ & $\begin{array}{l}\text { Predicted Low } \\
\text { Stress }\end{array}$ & Normal & Weird \\
\hline 1.89 & 2.02 & 1.58 & 3.85 \\
\hline
\end{tabular}

was performed on the scores for the experimental sentences. No significant difference was found between the two conditions. The experimental sentences were then compared with the filler sentences. Since there were, in all, 40 experimental sentences, however, and only half as many filler sentences, 10 in each category, only the predicted low-stress experimental items were tested against the two types of fillers. (The predicted lowstress category was chosen as it had the higher mean rating, i.e., was closer to the mean for the weird sentences and further from the mean for the normal sentences, whereas the most desirable result of this analysis would be a significant difference between the experimental items and the weird fillers, but no difference between the experimental items and the normal fillers.) Since there were unequal numbers in the two categories, an unweighted mean analysis of variance was again performed. The difference between the predicted lowstress items and the weird filler items was highly significant, $F(1,28)=44.01, p<.001$. The difference between the predicted low-stress items and the normal fillers was also significant, but to a lesser extent, $F(1,28)=6.78, p<.025$. Thus it would appear that the experimental items did not sound absolutely normal; however, there was no significant difference between the two experimental conditions as to the oddness of their contour.

The degree of correlation between reaction time scores for the spliced sentences and the rated oddness of these sentences was also tested. A low positive correlation was found between high-rated oddness and longer reaction time $\left(r_{s}=+.315\right)$, but this narrowly missed significance at the .05 level. Thus it appears extremely unlikely that the abnormality of the spliced contours determined the reaction time result.

\section{DISCUSSION}

The results of this experiment offer some solid evidence for the prediction of upcoming stress locations as an integral part of sentence understanding. An acoustically identical word was perceived faster when embedded in a suprasegmental context which predicted that it would bear high stress than in a context which predicted that it would bear reduced stress. Obviously, this difference cannot be ascribed to superior intelligibility of stressed words. Instead, the contextual variations must be the source of the difference. Exactly the same sequence of words preceded the target item in each case; the only difference lay in the intonation contour. We may therefore assume, since the two contexts produced different effects, that the sentence processing mechanism made use of the cues offered by the intonation contour. The difference between reaction times in the predicted high-stress context and in the predicted low-stress context was of the same nature as the difference previously observed between highand low-stress items; therefore, we assume that the use that the sentence processor made of intonational cues was to determine the relative stress level of items in the string before these items actually occurred.

Since no spectrographic analyses were made, it will be assumed that the cues offered by the intonation contour were of at least two kinds: variations in the relative duration of the words preceding the target item and variations in the pitch contour. ${ }^{1}$ The relative importance of pitch and durational cues in enabling the prediction of upcoming stress-whether, for example, either is a necessary and/or sufficient cueremains a subject for future research, since the present experiment offers no information on that point. What has been shown is that the processing of these cues allows the prediction of upcoming stress locations in normal sentence comprehension.

Before drawing further inferences from this finding, what of the remaining results of the experiment? The difference between high- and low-stress items in the unspliced condition was nearly three times as large as the difference between the predicted high- and predicted low-stress items in the spliced condition. We must assume this additional difference to be due to the acoustic differences which, in this case, obtained between high- and low-stress words. This leads us to reject the somewhat counterintuitive finding of Shields et al. that acoustic differences have no effect at all. The effect of such differences is large, and presumably accounts for nearly two-thirds of the observed differences in reaction time to stressed vs. unstressed words in previous experiments. However, the remaining one-third, still a highly significant difference, is not due to acoustic variation.

Further, the results of the "oddness" ratings allow us to reject the notion that the difference between the predicted high- and predicted low-stress items is due to effects of acoustic discontinuity introduced by the splicing intervention. Reduced stress in a predicted high-stress position does not appear to sound any more or less anomalous than heightened stress in a predicted low-stress position. ${ }^{2}$

We are led, then, to the inescapable conclusion that prediction of upcoming stress locations is an integral part of the sentence comprehension process. Further, this prediction is undertaken for the apparent end of focusing attention on the highly stressed portions of the sentence-since these are perceived more rapidly than the less stressed portions. At this point we may ask: what is it exactly that the processing mechanism finds when it directs attention to the location of a stress? 
As was observed above, it finds a segment which is lengthened, higher pitched, and of somewhat greater intensity than surrounding segments. The most receptive parts of the speech signal for changes in length, pitch, and intensity are, of course, the voiced portions. Bassically, a stressed segment is a vowel. ${ }^{3}$ Since a word may contain several vowels, the part of a stressed word which actually bears the stress may comprise only a small fraction of the word. (In a polysyllabic word in isolation, one syllable-not necessarily in a particular position in the word-bears heavier stress than the others, and it is that syllable which receives the sentential stress which may fall on the word when it occurs in running speech.)

A "stressed item," then, is at best part of a word, the vowel in the stressed syllable of a word which may have one or many syllables. It is probably fair to assume, however, that the entire stress-bearing syllable is reasonably intelligible, although the stress is actually applied to the vocalic nucleus, since a clearly articulated vowel provides clear transitional cues to preceding and following consonants.

Presumably the focusing of attention on stressed items is not without purpose; it must be assumed to facilitate the process of sentence understanding. If we suppose that the identification of a stressed syllable is of specific assistance to one or more of the necessary operations comprising the comprehension of a sentence, some interesting possibilities arise. Consider, for example, the hypothesis that the mental lexicon is so arranged that one (or the) primary principle by which a word is classified is the nature of its stressed syllable (or the vocalic nucleus of that syllable). ${ }^{4}$ When a stressed syllable is identified, then, the sentence processor can begin immediately to locate in the mental lexicon the word of which it is a part, using information not only about the stressed syllable itself but about the number of unstressed syllables immediately before and after it. A number of possible matches might be found and compared with the (incomplete) information about the phonetic structure of the unstressed syllables before a choice was made. Once the match was decided upon, the word boundaries could be drawn and preceding and succeeding words looked up.

If the lexical lookup operation is indeed of this nature, then it would make eminent strategic sense for the processing mechanism to predict the locations of upcoming stresses. Note that certain important consequences for a model of the sentence comprehension process would follow from acceptance of this hypothesis. We have suggested that stressed portions of the speech signal might be processed earlier than unstressed portions which actually precede them. Since some of these unstressed portions may themselves comprise words, it would follow that the words of an incoming sentence would not necessarily be processed in left-toright order. Further, the operations of phonetic identification, segmentation, and lexical lookup would not necessarily occur in that order for a given word, since segmentation, or identification of the boundaries of the word, could be consequent upon location of a match in the lexicon.

Although this view of the mental lexicon is entirely speculative, some indirect evidence does exist which supports it. Persons in the "tip-of-the-tongue" state often produce words which, while unrelated in meaning to the target word, reproduce correctly its stress pattern and the nature of its stressed syllable (Brown \& McNeill, 1966). Studies of hearing errors (Bond, 1973; Games \& Bond, 1975) show that the phonetic segments most frequently erroneously identified are the unstressed portions of a word or phrase irrespective of their position in it, whereas the vowel in the stressed syllable is usually identified correctly. Both of these findings indicate that among a word's closest neighbors in the mental lexicon are words with the same stressed syllable.

Recent work on speech errors involving substitution of semantically unrelated words (Fay \& Cutler, 1975) has provided strong evidence for the existence of a single mental lexicon for the purposes of both speech production and comprehension, arranged for optimal utility in the comprehension process-i.e., arranged by sound properties, such that any word's nearest neighbor in the lexicon is the word which most sounds like it, irrespective of meaning. Although Fay and Cutler described the principle of arrangement as a listing by left-to-right phonemic structure, the altemative offered above does not contradict the evidence they presented-words which sound alike would, under the stressed-syllable principle, still be listed together in the lexicon. In fact, the Fay and Cutler data provide a small measure of support for the present alternative, in that their targeterror pairs tend to be somewhat more alike in the stressed syllable than they are in the initial syllable, but the number of pairs on which this comparison was performed (obtained after excluding from the analysis monosyllables and words with stress on the initial syllable) was unfortunately too small for this support to be considered anything more than tentative.

\section{REFERENCES}

Bolinger, D. L. Contrastive accent and contrastive stress. Language, 1961, 37. 83.96.

BewD, Z. S. Perceptual errors in ordinary speech. Zeitschrift fiï Phonetik, 1973, 26, 691-694.

Brown, R., \& MCNeIll, D. The "tip of the tongue" phenomenon Journal of Verbal Learning and Verbal Behavior. 1966. 5. $325-337$

CLARK, H. H. The language-as-fixed-effect fallacy: A critique of language statistics in psychological research. Journal of Verbal Learning and Verbal Behavior, 1973, 12. 335-359.

Cutler, A., \& Foss, D. J. On the role of sentence stress in sentence processing. Language \& Speech, in press.

FAY, D., \& Cutrer, A. You have a dictionary in your head, not a thesaurus. Texas Linguistic Forum, 1975, 1, 27-40. 
Foss. D. J. Decision processes during sentence comprehension: Effects of lexical item difficulty and position upon decision times. Journal of Verbal Learning and Verbal Behavior. 1969, 8, 457-462.

Foss, D. J. Some effects of ambiguity upon sentence comprehension. Journal of Verbal Learning and Verbal Behavior. 1970. 9. 699-706.

GARnes. S., \& Bond, Z. S. Slips of the ear: Errors in perception of casual speech. Papers from the Eleventh Regional Meeting, Chicago Linguistic Society, 1975.

KLATr. D. The duration of [s] in English words. Journal of Speech \& Hearing Research, 1974, 17, 51-63.

KLATT. D. Voice onset time, frication, and aspiration in word-initial consonant clusters. Joumal of Speech \& Hearing Research, 1975, 18, 686-706.

Lehiste, I. Suprasegmentals. Cambridge, Mass: M.I.T. Press. 1970.

LIEBERMAN. P. Some effects of semantic and grammatical context on the production and perception of speech. Language and Speech, 1963, 6, 172-187.

MARTIN. J. G. Rhythmic (hierarchical) versus serial structure in speech and other behavior. Psychological Review. 1972. 79. 487-509.

Meltzer. R. H.. Martin. J. G.. Mills. C. B.. Imhoff, D. L.. \& ZOHAR. D. Reaction time to temporally-displaced phoneme targets in continuous speech. Journal of Experimental Psycholog: Human Perception and Pertormance. 1976; 2. 277-290.

Shieldos. J. L.. McHugh, A., \& Martin. J. G. Reaction time to phoneme targets as a function of rhythmic cues in continuous speech. Joumal of Experimental Psychology. 1974. 102. 250-255.

WINER. B. J. Statistical Principles in Experimental Design. New York: McGraw-Hill. 1971.

\section{NOTES}

1. Although no spectrographic analyses of the sentences were undertaken, the total duration of each sentence from sentence onset to onset of the target phoneme was measured. While no sentence produced identical measurements for the high and low versions, the mean duration of the high- and lowstress sentences differed by less than $1 \mathrm{msec}$. Thus, while the relative duration of the segments comprising the portion of the sentence prior to the target was certainly different for highvs. low-stress sentences, it was not the case that either high- or low-stress sentences were systematically longer (allowing, for example, more time for processing the pretarget portion).

2. Meltzer. Martin. Mills. Imhoff. and Zohar (1976), reporting recent work on the effect of splicing interventions. maintain that any such intervention will have the effect of raising reaction times, over and above any effects of experimental manipulations. For the present study, their prediction would therefore be that the reaction times to the spliced sentences within each condition should be longer than the reaction times to the corresponding unspliced sentences, even for the low-stress items (although the spliced predicted-low-stress target is actually of somewhat higher stress, and hence acoustically more intelligible, than the unspliced low-stress target). This was, in fact, the case when the results were averaged over the three separate administrations (see Table 1), although it did not obtain for each administration separately. The "oddness" ratings collected in the present study confirm that the splicing intervention did not affect the experimental conditions differentially.

It should further be noted that a test of rank-order correlation performed on the reaction time scores of the spliced sentences and of the unspliced versions of the same sentences showed a high positive correlation $\left(r_{s}=+.727, p<.001\right)$.

3. But there is evidence that consonants in prestressed position are of somewhat greater duration (Klatt, 1974, 1975).

4. Note that although each word has one and only one stress pattern in isolation, words with differing stress patterns are often very closely related, e.g., consist only of the same stem plus differing sets of bound morphemes. "Telegraph," "telegraphic." "telegraphy" are cases in point. One might expect that such words should be close lexical neighbors. This is possible in this model if one assumes that several kinds of adjacency are possible. Words with a common stressed syllable and many other sounds in common are adjacent in the sense that they are twigs on the end of the same branch of the same trunk-i.e., they share a large part of their classification structure. However, words which differ only in morphemic affixes may still have their meaning listed at the same point, i.e., may be adjacent at the point at which their classification structures terminate although these begin elsewhere. After all, twigs from one tree may be entwined with twigs from another tree.

(Received for publication November 24, 1975; revision received April 25, 1976.) 